Table 2. TIMnd DEschits: CoMparison of WAXED SkI WITH NEW SkI OB POLYTETRAFLOORETH YLIGNH (a) Crystalline spring snow ; sir temperature $5^{\circ} \mathrm{C}$.; snow ternperature
$0^{\circ} \mathrm{C}$.; gentle slope of length $700 \mathrm{ft}$. (tracked)

\begin{tabular}{|c|c|c|}
\hline Weight on ski (lb.) & $\begin{array}{c}\text { Time of descent (sec.): } \\
\text { Conventional ski with } \\
\text { Norwegian wax and } \\
\text { paraffin }\end{array}$ & $\begin{array}{c}\text { New ski of polytetra. } \\
\text { fluorethylene }\end{array}$ \\
\hline 168 & 61 & 42 \\
\hline 140 & 83 & 54 \\
\hline
\end{tabular}

(b) New snow fallen a few hours previously; air temperature $10^{\circ} \mathrm{C}$. snow temperature $0^{\circ} \mathrm{C}$.; unloaded ski (tracked)

\begin{tabular}{|c|c|c|}
\hline Detalls of slope & $\begin{array}{c}\text { Time of descent (sec.) } \\
\text { Conventional ski, } \\
\text { with ski lacquer }\end{array}$ & $\begin{array}{c}\text { New ski of polytetra- } \\
\text { fluorethylene }\end{array}$ \\
\hline $\begin{array}{c}\text { Gentle, } 20 \mathrm{~m} \text {. long } \\
\text { Steeper, 30 m. long }\end{array}$ & 10 & 6 \\
\hline
\end{tabular}

influenced by the contact angle which the water film makes with the surface. With polytetrafluorethylene this contact angle is very high $\left(126^{\circ}\right)$, and it remains high even after prolonged sliding on snow. This is appreciably higher than the contact angle observed on waxed surfaces which have been rubbed on snow. The experiments suggested that polytetrafluorethylene might have interesting possibilities as a skiing surface, and several pairs of skis have been coated with it and tried out in the Alps under a variety of snow conditions during the past three years. Some timed straight-run descents under controlled conditions have also been made. The results are quite striking, some typical experiments being illustrated in Table 2 .

It is clear that skis of polytetrafluorethylene are considerably faster under these conditions. They are easy to ski on, because they give a low uniform friction even when running on patchy snow. They should have useful applications not only to skiing but also to sledging and to aircraft landing, particularly on very cold, very wet or difficult snow and ice.

F. P. BowDEN

Physics and Chemistry of Surfaces,

Department of Physical Chemistry,

University of Cambridge.

' Bowden, F. P., and Ridler, K. E. W., Proc. Roy. Soc., A, 151, 610 (1936).

- Beams, J. W., J. Washington Acad. Sci., 37, 221 (1947).

"Reynolds, O., "Paper on Mechanical and Physical Subjects, 2" (Cambridge University Press, 1901).

- Bowden, F. P., and Hughes, T. P., Proc. Roy. Soc., A, 172, 280 (1939).

' Bowden, F. P., Proc. Roy. Soc., A, 217, 462 (1953). See also Hawksley Lecture, J'. Inst. Mech. Eng., 2, 86 (1955).

-Bowden, F. P., and Tabor, D., "Friction and Lubrication of Solids" (Oxford: C̈larendon Press, 1954).

\title{
SOLAR ECLIPSES AND THE IONOSPHERE
}

ONOSPHERIC measurements at the time of solar eclipses may be expected to yield useful information on problems such as the nature of the ionizing radiation and its distribution on and outside the visible solar disk, on theories of the formation of the ionosphere and of recombination processes within the various layers. It has long been established that the primary ionizing source of the $E$ and $F 1$ layers of the ionosphere is solar ultra-violet light. This conclusion is amply confirmed both by long-term measurements and by results obtained during solar eclipses. How. ever, in the case of region $F 2$ the source is less clearly defined; eclipse effects in this region are often complex and the interpretation of the observations ambiguous. Certain aspects of eclipse phenomena in all the ionospheric regions were discussed at a symposium on "Solar Eclipses and the Ionosphere" held in the rooms of the Royal Society, London, during August 22-24. The symposium was organized by the Mixed Commission on the Ionosphere and sponsored by the International Council of Scientific Unions. Some twenty-five British and fifty-five overseas delegates participated.

Opening the symposium, Mr. J. A. Ratcliffe presented a general survey of ionospheric eclipse phenomena and discussed the eclipse effects to be expected in a simple Chapman-type layer. Eclipse results for region $E$ have been reconciled with the predictions of simple theory by supposing that the process of electron loss is recombination-like with an effective coefficient $\alpha^{\prime} \simeq 10^{-8} \mathrm{~cm} \cdot{ }^{8} \mathrm{sec} .^{-1}$. In most cases it has also been necessary to suppose that the intensity of the ionizing radiation is not uniform over the solar disk, and furthermore that a measurable fraction originates outside the visible disk. In the case of region $F^{\prime} 1$, eclipse estimates of $\alpha^{\prime}$ lie between 5 and $9 \times 10^{-9} \mathrm{~cm} . .^{3} \mathrm{sec} .^{-1}$, and for this region there is again evidence for enhanced radiation from the same areas which contribute to the $E$-layer ionization. In the
F'2 layer complex eclipse effects are often observed, and here it is clear that consideration must be given to the influence of movements in the layer during the eclipse. An interesting phenomenon which has received attention during recent eclipses is the temporary appearance of a new critical frequency between the $F^{1} 1$ and $F 2$ critical frequencies. It has been suggested that this might correspond-to a separate layer, the so-called $F 11$ layer, due to some special process of electron production. However, it can be shown that if the electron loss coefficient decreases upwards, then the appearance, during an eclipse, of a point of inflexion on the $h^{\prime} f$ curve between the $F 1$ and $F 2$ layer critical frequencies is to be expected.

Mr. C. M. Minnis discussed eclipse results for region F'2 obtained at Khartoum, Singapore, Slough and Inverness during eclipses of 1952, 1954 and 1955 and stressed the desirability of always computing the electron density/true height profile, since deductions based only on critical frequency variations can be misleading.

Prof. S. Chapman outlined the development of ideas concerning a possible corpuscular eclipse effect in the ionosphere. The observed delay of about one day between certain solar disturbances and terrestrial magnetic storms indicates that the solar particles have an average speed of about 1,000 miles/sec., and for this speed the corpuscular eclipse might be expected to occur about two hours before the optical eclipse. This difference in time would, of course, result in a considerable difference in position on the earth between the optical and corpuscular eclipses. As far back as 1919 Chapman and Milne pointed out that a speed of 1,000 miles/sec. seemed quite inadequate for particles to reach the $100-\mathrm{km}$. level, and it is now believed that if there are any corpuscular eclipse effects these may be expected to be weak and to affect only a high layer in the ionosphere. Prof. Chapman also outlined the theory of the effect of an 
eclipse on the Earth's magnetic field. The reduction in the electron and ion content of the ionosphere during a solar eclipse reduces the conductivity; this modifies the electric current intensity and hence affects the transient variations in the earth's field. In general, the magnetic effect of an eclipse may be expected to be quite small-the precise magnitude and nature will depend on the location of the observing site with respect to the electric current systems in the ionosphere. At stations near the foci of these systems the eclipse effect may be expected in the vertical force, whereas in equatorial latitudes it should appear in the north horizontal force.

Recent successful measurements of geomagnetic effects during a solar eclipse were described by $\mathrm{Mr}$. J. Egedal. Records of magnetic declination from fourteen observatories in Europe showed small but quite definite effects during the eclipse of June 30 , 1954. The magnitude was of the order to be expected if the ionospheric currents concerned flowed near the level of region $E$. Dr. T. Nagata presented similar results for observations in Japan during the eclipse of May 9, 1948. In this case it was found that the magnitude of the changes in the east component were consistent with calculations based on the observed eclipse decrease in electron density in the $E$-layer.

One session was devoted to a discussion of present solar knowledge with particular reference to ionospheric studies. Prof. C. W. Allen showed that longperiod ionospheric data for regions $E$ and $F$ require a quiet component from the whole Sun together with a component associated with centres of activity. Dr. M. A. Ellison discussed some ionospheric effects of solar flares, and Prof. D. R. Bates presented a short summary of present views concerning the theory of layer formation. The $D$ layer is now believed to be due to photoionization of nitric oxjde by Ly $\alpha$, the $E$ layer to X-rays centred mainly near $40 \mathrm{~A}$., and the $F 1$ and $F 2$ layers partly to longer $\mathrm{X}$-rays and partly to ultra-violet ionization of atomic oxygen. Mr. M. Ryle and Dr. J. P. Hagen respectively presented summaries of knowledge concerning the solar corona and chromosphere as deduced from solar radio noise studies.

A session on recombination in the ionosphere was introduced by Prof. D. R. Bates, who emphasized that recombination takes place through a complex variety of processes. For the higher layers the basic mechanism is probably dissociative recombination. In the case of the $D$-layer the position is still open. To explain the very large recombination coefficients for the $D$-layer, the mechanism might be negative ion recombination, it might be ordinary dissociative recombination or the process might involve complex ions. Prof. J. Sayers briefly outlined laboratory experiments on recombination processes now in progress in the University of Birmingham.

Measurements of effective recombination coefficients deduced from solar eclipse ionospheric observations were presented by Mr. C. M. Minnis, Dr. O. Burkard and Dr. K. Rawer. Mr. Minnis showed how the effective recombination coefficients deduced from cclipse measurements depend critically on assumptions concerning the amount of residual radiation at totality. He also showed that although it is often difficult to give acourate estimates of $\alpha^{\prime} E$ and $\alpha_{F 1}^{\prime}$, it is possible, both from measurements of the normal diurnal variations in $f E$ and $f F 1$ and from eclipse observations, to obtain reliable estimates of the ratio $\alpha_{E}^{\prime} / \alpha^{\prime} F$. Dr. Rawer stressed the errors likely to be involved in evaluating effective recombination coefficients by applying an over-simplified theory to eclipse observations. The usual assumptions of monochromatic ionizing radiation, of an isothermal atmosphere and of simple recombination processes are not satisfied, and in presenting future ionospheric rosults he suggested that data should be given for parameters which are independent of any special theories. Dr. Burkard considered ionospheric data for the eclipse of February 25,1952 , and showed that if the results are interpreted not in terms of the usual simple theoretical equilibrium equation but in terms of a modified equation in which layer conditions are not assumed to be isothermal, then there is no necessity to assume a non-uniform distribution of the sources on the solar disk as had been done by other workers.

Mr. W. R. Piggott discussed eclipse observations of vertical-incidence ionospheric absorption at Ibadan in 1952 and showed that eclipse changes in the absorbing region and in regions $E$ and $F 1$ all appeared to indicate similar (irregular) distributions of the emission of the ionizing radiation from the Sun. Interesting eclipse observations on the lowest ionosphere were described in papers by $\mathrm{Dr}$. K. Weekes and by Dr. E. A. Lauter.

In the final session of the meeting, Dr. S. D. Gossner directed attention to some unusual geometrical conditions pertaining to the solar eclipse of October 23, 1957. These conditions result from the fact that the axis of umbra will pass near the surface of the Earth without actually touching it and the central lines will intersect the ionosphere twice. For vertical soundings this second intersection will take place in the general area of the Falkland Islands Dependencies.

The principal points which would appear to have arisen from discussions at this symposium are the following. In the case of region- $E$, some 10-15 per cent of the ionizing radiation comes from beyond the visible solar disk. The uncertainty concerning the actual magnitude of this residual radiation means that there is corresponding uncertainty about the effective recombination coefficients deduced from eclipse observations. In interpreting eclipse results it is also very important to consider what is the appropriate value for the index $m$ in the expression $f E \propto(\cos \chi)^{m}(\chi=$ solar zenith angle). In general, experimental results do not fit the value predicted by simple theory, namely $m=0 \cdot 25$, and during the discussions a number of possible causes for the observed variations in $m$ were brought to light. In the case of region $F 1$, the above difficulties are again all present. It is clear that, in eclipse observations on this region, changes in the electron density/true height profile and not merely changes in the critical frequency $f F 1$ must be considered. In the case of region $F 2$, similar profiles must also be calculated, and the discussions also clearly brought out the importance of movements in this region. Several speakers referred to the appearance of the $F 1 \frac{1}{2}$ trace during eclipse observations, and although some results suggested that this phenomenon is confined to places of low magnetic dip and to eclipses of large magnitude, it was not clear that this is always the case.

During the symposium, limitations of time only permitted the presentation of summaries of a few of the fifty papers submitted. These papers, together with the discussions, will shortly be published in a single volume by the International Union for Scientifio Radio.
W. J. G. BEYNON 